\title{
A Retrospective Analysis of 111 Cases of Pneumococcal Pneumonia: Clinical Features and Prognostic Factors
}

\author{
Satoshi Ikegame ${ }^{1}$, Kentaro Wakamatsu ${ }^{1}$, Hiroyuki Kumazoe ${ }^{1}$, Masayuki Kawasaki ${ }^{1}$, \\ Masaki Fujita ${ }^{2}$, Yoichi Nakanishi ${ }^{3}$, Mine Harada ${ }^{1}$ and Akira Kajiki ${ }^{1}$
}

\begin{abstract}
Objective Streptococcus pneumoniae is an important and common pathogen of acute pneumonia. The urinary pneumococcal antigen test has been increasingly used for the diagnosis of pneumococcal pneumonia, but there have been few studies on pneumococcal pneumonia after this test became prevalent. The present study was conducted to characterize the clinical features of pneumococcal pneumonia after the introduction of the urinary antigen test.

Methods We retrospectively analyzed 111 cases from 105 patients with pneumococcal pneumonia in our hospital between 2007 and 2010, and collected data regarding background characteristics, laboratory data, isolated bacteria, and clinical courses.

Results The cases analysed included 77 of community-acquired pneumonia (CAP), 21 of healthcareassociated pneumonia (HCAP), and 13 of hospital-acquired pneumonia (HAP). Penicillin-resistant Streptococcus pneumoniae was isolated in 12.5 and $55.6 \%$ of non-HAP and HAP cases, respectively. When the cases were divided into 5 groups according to A-DROP scores (0, 1, 2, 3 and 4 points), critical events occurred at $0,0,8.7,15.4$, and $75.0 \%$ in groups of cases of $0,1,2,3$, and 4 points, respectively. Similarly, critical events occurred at $66.7,4.7,3.8$, and $9.1 \%$ in groups of cases according to WBC counts of $<4,000,4,000$ $9,999,10,000-19,999$, and $\geq 20,000$ cells/ $\mu \mathrm{L}$, respectively.

Conclusion Most of the pneumococcal pneumonia cases occurred as CAP and were treated successfully. HAP cases were frequently penicillin resistant. Elevated A-DROP scores and decreased WBC counts were found to be predictive of critical events.
\end{abstract}

Key words: A-DROP, pneumococcal pneumonia, streptococcus pneumoniae, urinary pneumococcal antigen

(Intern Med 51: 37-43, 2012)

(DOI: 10.2169/internalmedicine.51.5847)

\section{Introduction}

Although acute pneumonia can be successfully treated with effective antibiotics, it is the fourth most frequent cause of death in Japan (1) and the world (2). Acute pneumonia is still an important infectious disease. Streptococcus pneumoniae is the leading microorganism causing communityacquired pneumonia (CAP) $(3,4)$ and lethal pneumonia (5), and should be also considered as an important cause of hospital-acquired pneumonia (HAP) (6).
In recent years, the urinary pneumococcal antigen test (7-9) has been increasingly used for diagnosing pneumococcal pneumonia. However, there have been few clinical studies on pneumococcal pneumonia after this test was introduced. In addition, the A-DROP system proposed by the Japanese Respiratory Society (JRS) in 2007 was not fully evaluated for its validation. To document or identify clinical characteristics of pneumococcal pneumonia and evaluate the usefulness of A-DROP system in pneumococcal pneumonia, we conducted a retrospective analysis of 111 cases (105 patients) based on the urinary pneumococcal antigen test and

\footnotetext{
${ }^{1}$ Department of Respiratory Medicine, National Hospital Organization Omuta National Hospital, Japan, ${ }^{2}$ Department of Respiratory Medicine, Faculty of Medicine, Fukuoka University, Japan and ${ }^{3}$ Research Institute for Diseases of the Chest, Faculty of Medicine, Kyushu University, Japan

Received for publication May 19, 2011; Accepted for publication September 6, 2011

Correspondence to Dr. Satoshi Ikegame, ikegame-s@oomuta-h.com
} 
culture results of $S$. pneumoniae. This led us to identify several important findings regarding the clinical features and prognosis of the disease.

\section{Materials and Methods}

\section{Inclusion criteria for pneumococcal pneumonia cases}

We screened 112 urinary pneumococcal antigen positive tests and 360 samples (from sputum, blood, pleural effusion, and bronchofiberscopy) that were culture-positive for $S$. pneumoniae between January 2007 and December 2010 according to our hospital database. BinaxNOW ${ }^{\circledR}$ S. pneumoniae (Binax, Inc, Scarborough, ME, USA) was used for the detection of urinary pneumococcal antigen. We established three diagnostic criteria: (i) bacteriological, (ii) clinical, and (iii) radiological. Bacteriological criteria required at least one factor from (a)-(d): (a) positive result for the urinary pneumococcal antigen test without pneumonia in the previous three months, (b) identification of $S$. pneumoniae cultured from sputum at $\geq 2+$ (equivalent to $10^{5} \mathrm{CFU} / \mathrm{mL}$ ), (c) identification of $S$. pneumoniae cultured from sputum at $\geq$ $1+$ (equivalent to $10^{3} \mathrm{CFU} / \mathrm{mL}$ ) with phagocytosed bacteria by Gram staining, and (d) identification of S. pneumoniae cultured from pleural effusion or blood. Clinical criteria included either fever $\geq 37.0^{\circ} \mathrm{C}$, cough, elevated white blood cell (WBC) counts $(\geq 10,000$ cells $/ \mu \mathrm{L})$, decreased WBC counts $(<4,000$ cells $/ \mu \mathrm{L})$, elevated CRP values $(\geq 0.30 \mathrm{mg} /$ $\mathrm{dL})$, or progression of hypoxemia $\left(\mathrm{SpO}_{2} \leq 90 \%\right.$ or $\mathrm{PaO}_{2} \leq$ 60 Torr). Radiological criteria retained either chest X-ray or computed tomography scans suggestive of pneumonia. The patients that met all three criteria were designated as a case of pneumococcal pneumonia. We selected 111 cases (events) from 105 patients that met the pneumococcal pneumonia criteria during the four-year study period and analyzed these cases (6 patients had a second bout of pneumococcal pneumonia).

\section{Data collection}

We collected the following data from the patients: background (gender, age, and comorbidities), laboratory test data at the onset of pneumonia (WBC counts, BUN levels, $\mathrm{Cr}$ levels, urinary pneumococcal antigen test results, results of bacterial culture, and oxygenation levels), physical examination findings (blood pressure, presence of dehydration, and level of consciousness), and clinical courses (duration of antibiotic therapy, requirement of mechanical ventilation, and mortality). The definition of CAP, healthcare-associated pneumonia (HCAP), and HAP were conformed to the ATS guideline (6). Nursing-home acquired pneumonia (NHAP) was defined as a pneumonia acquired in patients of nursinghome residence. NHAP is included by HCAP according to the ATS guideline. Resistance of isolated $S$. pneumoniae to penicillin, ceftriaxone (CTRX), ofloxacin (OFLX), and imipenem (IPM) were defined by National Committee for
Clinical Laboratory Standards (NCLLS) (10). PSSP, PISP, and PRSP were defined as MIC $\leq 0.063$, MIC $=0.12-1.0$, and MIC $\geq 2.0 \mu \mathrm{g} / \mathrm{mL}$, respectively. CTRX, OFLX and IPM resistance were defined as $\mathrm{MIC} \geq 4.0 \mu \mathrm{g} / \mathrm{mL}$, MIC $\geq 8.0$ $\mu \mathrm{g} / \mathrm{mL}$, MIC $\geq 2.0 \mu \mathrm{g} / \mathrm{mL}$, respectively. Drug sensitivity was checked by using the $S$. pneumoniae isolated before antibiotic treatment in our hospital. A-DROP scores were calculated by the sum of five risk factors (1: $\geq 70$ years old for males, or $\geq 75$ years old for females, 2 : BUN $\geq 21 \mathrm{mg} / \mathrm{dL}$, or presence of dehydration, 3: $\mathrm{SpO}_{2} \leq 90 \%$, or $\mathrm{PaO}_{2} \leq 60$ Torr, 4: Disturbance of consciousness, 5: Blood pressure $\leq$ $90 \mathrm{mmHg}$ ) (11). The study protocol was approved by the institutional review board of our hospital.

\section{Statistical analysis}

We compared frequencies of critical events (Table 3, 6-9, as defined by requirement of mechanical ventilation or death by pneumonia) and frequencies of penicillin-susceptible $S$. pneumoniae (PSSP), penicillin-intermediate S. pneumoniae (PISP), and penicillin-resistant S. pneumoniae (PRSP) (Table 5) with a chi-square test. We compared the duration of antibiotic therapy among the patients depending on A-DROP scores (Table $6,8,9$ ) with a t-test.

\section{Results}

Six of the 105 patients developed secondary pneumococcal pneumonia, and thus 111 cases in total were analyzed in this study. As shown in Table 1, 59 cases occurred in male patients and 52 occurred in female patients. The median age was 73 years old (ranging 15 to 97 years old) with 89 cases $(82 \%)$ occurring in patients over 60 years of age. Ten cases had diabetes mellitus, eight had malignancy (four of which were lung cancer), 36 had respiratory diseases other than lung cancer, and 14 had a swallowing disorder (according to medical records of our hospital during pneumonia). Nine cases were receiving systemic steroid therapy for their comorbidities, and four cases had been vaccinated for pneumococcal pneumonia.

Table 2 shows the bacteriological diagnosis in the cases of pneumococcal pneumonia. Seventy-two cases (64.9\%) were diagnosed by the urinary pneumococcal antigen test, $25(22.5 \%)$ by culture results only, and $14(12.6 \%)$ by both methods. Sputum was cultured in 67 of the 86 cases positive for the urinary pneumococcal antigen test. S. pneumoniae was isolated in 22 cases $(32.8 \%)$, but only 11 cases $(16.4 \%)$ met the bacteriological criteria $(\geq 2+$, or $\geq 1+$ with detection of phagocytosed bacteria by Gram staining).

Seventy-seven of 111 cases $(69.0 \%)$ were CAP and 60 of them were admitted to our hospital. Twenty-one of the cases (18.9\%) were HCAP and 20 of them were admitted to our hospital. Seven of the cases $(6.3 \%)$ were nursing homeacquired pneumonia (NHAP, which is included in HCAP) and all of them were admitted to our hospital. Thirteen of the cases $(11.7 \%)$ were HAP and all of them continued to stay in the hospital (three were transferred to our hospital 
Table 1. Characteristics of 111 Cases (105 Patients)

\begin{tabular}{lc}
\hline \multicolumn{1}{c}{ Characteristics } & No. of cases (\%) \\
\hline Gender: & $59(53.2 \%)$ \\
Male & $52(46.8 \%)$ \\
Female & 4 cases $(3.6 \%)$ \\
Vaccination & \\
Comorbidities & $10(9.0 \%)$ \\
Diabetes mellitus & $8(7.2 \%)$ \\
Malignancy & $9(8.1 \%)$ \\
Steroid therapy & $36(32.4 \%)$ \\
Respiratory disease & \\
(other than lung cancer) & $14(12.6 \%)$ \\
Swallowing disorder & $6(5.4 \%)$ \\
Neuromuscular disease & \\
Bronchotomy & \\
\end{tabular}

Median age was 73 years old, ranging from 15 to 97 years old.

*Includes three cases of amyotrophic lateral sclerosis, two cases of muscular dystrophy,

and one case of spinocerebellar degeneration. All six patients were bedridden due to

their diseases.

${ }^{\dagger}$ Patient received bronchotomy for amyotrophic lateral sclerosis-induced respiratory

failure.

Table 2. Bacteriological Diagnosis

\begin{tabular}{cc}
\hline Method & Cases $(\%)$ \\
\hline Urinary pneumococcal antigen test & $72(64.9 \%)$ \\
Bacterial cultures & $25(22.5 \%)$ \\
Both & $14(12.6 \%)$ \\
\hline
\end{tabular}

for pneumonia). We defined a critical event as requirement of mechanical ventilation or death by pneumonia as a critical event, and relationships between critical events and pneumonia type were analyzed (Table 3). The frequency of critical events in CAP was 3.9\% (3/77). In contrast, the frequency of critical events in NHAP was elevated to $28.6 \%$ $(2 / 7)$, but statistically not significant $(p=0.05)$. The frequency of deaths was significantly higher in NHAP than in CAP $(1.3 \%$ in CAP vs. $28.6 \%$ in NHAP; $\mathrm{p}=0.02)$.

Next, we investigated the frequency of mixed bacterial infection in 89 pneumococcal pneumonia cases in which sputum or bronchofiberscopic samples were obtained for bacterial culture. Using similar bacteriological criteria as pneumococcal pneumonia, we diagnosed 11 cases of mixed infection (12.4\%): four cases with Haemophilus influenzae, three cases with Pseudomonas aeruginosa, three cases with Escherichia coli, and two cases with Moraxella catarrhalis (Table 4). The relationship between mixed infection and poor clinical outcome was not clear in this study because there were no critical events in the cases of mixed infection.

S. pneumoniae was isolated in 50 cases (including cases in which only a small amount of bacteria was isolated from sputum culture), and drug resistance was tested in 49 of those cases. PRSP was identified in $20.4 \%$ of the cases and the isolation was increased up to $55.6 \%$ in the cases of HAP (Table 5), which was significantly more frequent than that in non-HAP cases $(12.5 \%, \mathrm{p}=0.01)$. The proportion of OFLX resistance was also higher in HAP cases than in non-HAP cases $(55.6 \%$ in HAP vs. $5.7 \%$ in non-HAP, $p=0.002)$. On the other hand, the proportion of CTRX resistance (total:
6.1\%, HAP: $11.1 \%$, non-HAP: $5.7 \%, \mathrm{p}=0.46)$ and IPM resistance (there was no IPM resistant $S$. pneumoniae in this study) were lower, and there was no difference between HAP and non-HAP cases. There were no critical events in 10 cases in which PRSP was isolated; the relationship between PRSP isolation and poor clinical outcome was not shown.

We were able to evaluate A-DROP scores, which is a criterion proposed by the JRS for assessing the severity of CAP (11), in 102 cases. The frequency of critical events was increased along with A-DROP scores (critical events occurred at $8.7,15.4$, and $75.0 \%$ in cases of A-DROP scores of 2,3 , and 4 points, respectively), whereas there were no critical events in cases of A-DROP scores of 0 or 1 (Table 6). On the other hand, A-DROP scores were not correlated with the duration of therapy. We then analyzed the relationship between positive urinary pneumococcal antigen tests and A-DROP scores. There was no statistically significant difference in the incidence of positive urinary pneumococcal antigen tests among A-DROP score groups (Table 7).

We performed a similar analysis for CRP levels (Table 8). The critical events occurred in $42.9 \%$ of cases with CRP levels $\geq 30 \mathrm{mg} / \mathrm{dL}$, which was statistically significantly more frequent $(\mathrm{p}<0.01)$ than those with $\mathrm{CRP}<10 \mathrm{mg} / \mathrm{dL} \quad(3.8 \%)$. However, the critical events also occurred in cases with CRP $<10 \mathrm{mg} / \mathrm{dL}$ and $10.0-19.9 \mathrm{mg} / \mathrm{dL}$. We also observed a statistically significant correlation between elevated CRP levels and prolonged periods of antibiotic therapy (Table 8).

Finally, we investigated the relationship between WBC counts and the duration of therapy (Table 9). Although only three cases showed decreased WBC counts $(<4,000$ cells/ $\mu \mathrm{L})$, their duration of therapy was prolonged $(21.3 \pm 5.8$ days) and the critical events occurred at an extremely high frequency $(66.7 \%)$ compared to cases with normal WBC counts $(4,000-9,999$ cells $/ \mu \mathrm{L}$ ). Multivariate analysis (logistic regression analysis) including A-DROP scores, decreased WBC counts, and CRP levels revealed that elevated A- 
Table 3. Pneumonia Type and Outcome

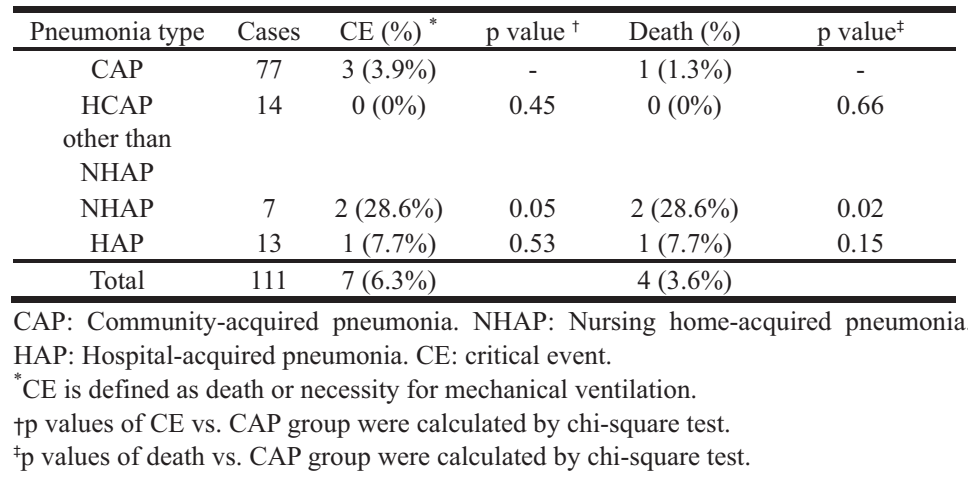

Table 4. Bacteria Cultured in Mixed Infections

\begin{tabular}{cc}
\hline 89 pneumococcal pneumonia cases & Cases (\%) \\
\hline Haemophilus influenzae & $4(4.5 \%)$ \\
Pseudomonas aeruginosa & $3(3.4 \%)$ \\
Escherichia coli & $1(1.1 \%)$ \\
Escherichia coli + Enterococcus faecalis & $1(1.1 \%)$ \\
Moraxella catarrhalis & $1(1.1 \%)$ \\
Moraxella catarrhalis + Streptococcus mitis & $1(1.1 \%)$ \\
\hline Total & $11(12.4 \%)$ \\
\hline
\end{tabular}

DROP scores $(\mathrm{p}<0.01)$ and decreased WBC counts $(\mathrm{p}<0.01)$ were independently related to the critical events. The duration of therapy also tended to be prolonged in cases with increased WBC counts ( $\geq 20,000$ cells $/ \mu \mathrm{L})$, but the frequency of the critical events was not increased.

\section{Discussion}

In this study, we investigated the clinical features and prognostic factors of pneumococcal pneumonia not only in cases of CAP, but also in HCAP and HAP cases. The frequency of PRSP was higher in HAP than in CAP and HCAP, while the risk of death was higher in NHAP than in CAP. The A-DROP system was useful in predicting critical events, and decreased WBC counts were an independent predictor of the critical events. It is very important for successful treatment to determine the patients with a bad prognosis and drug resistance, and to treat such patients appropriately and intensively.

We confirmed that the urinary pneumococcal antigen test greatly contributed to the diagnosis of pneumococcal pneumonia in the general hospital. There were 67 urinary pneumococcal antigen positive cases in which sputum cultures were also checked. S. pneumoniae was not isolated in 44 cases $(65.7 \%)$, and isolated in 23 cases (11 cases of which met our bacteriological criteria). This finding is consistent with a previous study reported by Gutiérrez et al (12), in which 69 of 88 urinary antigen positive cases $(78 \%)$ were culture negative. There are several factors that may reduce the sensitivity of culture results, such as a preceding antibiotic therapy and quality of sputum samples. Therefore, the urinary antigen test is an important diagnostic tool for pneu- mococcal pneumonia.

It is interesting that the incidence of swallowing disorder was remarkably high as a comorbidity (12.6\% of patients) (Table 1). Our survey depended on the medical records, so only evident cases were regarded as swallowing disorder. This prevalence may be influenced by definition, patient age distribution, and hospital characteristics. Accordingly more studies are needed to evaluate the relationship between swallowing disorders and pneumococcal pneumonia. El-Solh et al (13) reported that the causative agents of aspiration pneumonia include aerobic bacteria such as $S$. pneumoniae, Staphylococcus aureus, and Klebsiella pneumoniae. We should not underestimate the involvement of S. pneumoniae when treating aspiration pneumonia.

Fuse et al (14) reported that the frequency of positive urinary pneumococcal antigen tests was not changed regardless of the A-DROP scores. In the present study, the frequency of positive urinary pneumococcal antigen tests was slightly increased in association with A-DROP scores $(86.2 \%$ in the A-DROP 0 points group and $93.8 \%$ in cases with A-DROP scores $\geq 3$ points, Table 6 ), but it was not statistically significant. Urinary pneumococcal antigen was efficiently detected, even in the mild cases.

When analyzing prognosis according to a pneumonia type, the frequency of death was significantly higher in NHAP than in CAP (Table 3). NHAP has been reported to have a poor prognosis (15), and we observed similar results in our study of pneumococcal pneumonia. The age of NHAP patients $(76.7 \pm 8.6$ years old $)$ was higher than that of CAP $(69.3 \pm 15.0$ years old), but it was similar to HCAP other than NHAP $(80.0 \pm 7.6$ years old $)$. Therefore the reason for the poor prognosis in NHAP might reside in the decreased ability of daily life (ADL). It is possible to predict the critical events by using the A-DROP system, but pneumococcal pneumonia in nursing homes should be treated intensively in the hospital. NHAP was included in HCAP in the ATS guideline (6). The present data regarding HCAP and NHAP was limited in number, but it suggested the possibility of difference in the characteristics between NHAP and HCAP.

The incidence of resistance of $S$. pneumoniae to penicillin, one of the primarily-selected antibiotics for treating 
Table 5. Susceptibility of Streptococcus pneumoniae Isolates to Penicillin G, Ceftriaxone, Ofloxacin, and Imipenem

\begin{tabular}{ccccc}
\hline & Total & HAP & Non-HAP & p value $^{*}$ \\
\hline PSSP & $17(34.7 \%)$ & $2(22.2 \%)$ & $15(37.5 \%)$ & 0.47 \\
PISP & $22(44.9 \%)$ & $2(22.2 \%)$ & $20(50 \%)$ & 0.16 \\
PRSP & $10(20.4 \%)$ & $5(55.6 \%)$ & $5(12.5 \%)$ & 0.01 \\
\hline CTRX resistance & $3(6.1 \%)$ & $1(11.1 \%)$ & $2(5.0 \%)$ & 0.46 \\
\hline OFLX resistance $^{\S}$ & $7(15.9 \%)$ & $5(55.6 \%)$ & $2(5.7 \%)$ & $<0.01$ \\
\hline IPM resistance & $0(0 \%)$ & $0(0 \%)$ & $0(0 \%)$ & 1.00 \\
\hline Total & 49 & 9 & 40 &
\end{tabular}

HAP: Hospital-acquired pneumonia. Non-HAP: Community-acquired pneumonia and healthcare-associated pneumonia. PSSP: Penicillin-susceptible Streptococcus pneumoniae. PISP: Penicillin-intermediate Streptococcus pneumoniae. PRSP: Penicillin-resistant Streptococcus pneumoniae. CTRX: ceftriaxone. OFLX: ofloxacin. IPM: imipenem

${ }^{*}$ Frequency in HAP vs. non-HAP group compared by chi-square test.

${ }^{\S}$ OFLX sensitivity was checked in 9 HAP cases and in 35 non-HAP cases.

Table 6. A-DROP Score and Clinical Course

\begin{tabular}{|c|c|c|c|c|c|}
\hline A-DROP & Cases $(\%)$ & $\begin{array}{c}\text { Critical events } \\
(\%)\end{array}$ & p value ${ }^{*}$ & $\begin{array}{c}\text { Duration of } \\
\text { therapy } \\
(\text { mean } \pm \text { S. D. })^{\dagger}\end{array}$ & p value ${ }^{\ddagger}$ \\
\hline 0 points & $34(33.3 \%)$ & $0(0 \%)$ & - & $13.3 \pm 8.4$ days & - \\
\hline 1 point & $28(27.5 \%)$ & $0(0 \%)$ & 1.00 & $14.7 \pm 8.1$ days & 0.53 \\
\hline 2 points & $23(22.5 \%)$ & $2(8.7 \%)^{\S}$ & 0.15 & $15.3 \pm 5.9$ days & 0.35 \\
\hline 3 points & $13(12.7 \%)$ & $2(15.4 \%)^{9}$ & 0.07 & $15.7 \pm 4.1$ days & 0.34 \\
\hline 4 points & $4(3.9 \%)$ & $3(75.0 \%) \#$ & $<0.01$ & & \\
\hline 5 points & $0(0 \%)$ & - & - & & \\
\hline Total number & 102 cases & 7 cases & & 98 cases & \\
\hline $\begin{array}{l}\text { p values vs. } 0 \mathrm{p} \\
\text { Cases in which } \\
\text { p values vs. } 0 \mathrm{p} \\
\text { All cases were } \\
\text { Death occurred } \\
\text { Death occurred }\end{array}$ & $\begin{array}{l}\text { ints group cal } \\
\text { death occurred } \\
\text { ints group cal } \\
\text { reated success } \\
\text { in all cases. } \\
\text { in two cases. }\end{array}$ & $\begin{array}{l}\text { llated by chi-squ } \\
\text { liminated from a } \\
\text { lated by t-test. } \\
\text { lly. }\end{array}$ & $\begin{array}{l}\text { test. } \\
\text { lysis. }\end{array}$ & & \\
\hline
\end{tabular}

Table 7. A-DROP Score and Urinary Pneumococcal Antigen Test Results

\begin{tabular}{ccc}
\hline A-DROP & Positive cases / total cases $(\%)$ & p value $^{*}$ \\
\hline 0 points & $25 / 29(86.2 \%)$ & - \\
1 point & $23 / 25(92.0 \%)$ & 0.67 \\
2 points & $18 / 20(90.0 \%)$ & 0.96 \\
$\geq 3$ points & $15 / 16(93.8 \%)$ & 0.64 \\
\hline Total number & $81 / 90(90.0 \%)$ & \\
\hline
\end{tabular}

" p values vs. 0 points group calculated by chi-square test.

Five cases were eliminated from analysis because A-DROP scores could not be calculated.

pneumococcal pneumonia, was found to be $12.5 \%$ in nonHAP cases in this study, which was comparable to that reported in a previous study conducted in Japan (16). On the other hand, penicillin-resistant isolates were increased up to $55.6 \%$ in HAP cases (Table 5). Hospitalization is a risk factor of multidrug resistant bacterial pneumonia (6). Thus, pneumococcal pneumonia in hospitals should be treated as PRSP because we found that HAP cases with S. pneumoniae were frequently resistant to penicillin G (MIC $\geq 2 \mu \mathrm{g}$ / $\mathrm{dL})$. Recently the new criteria of penicillin resistance regarding S. pneumoniae was proposed by CLSI (17). The proportion of PRSP (MIC $\geq 8 \mu \mathrm{g} / \mathrm{dL}$ ) may be reduced according to this strict criteria, but we could not assess by this criteria, because the upper limit of MIC in our study was $2 \mu \mathrm{g} / \mathrm{dL}$. To summarize Table 3 and Table 4, most pneumococcal pneumonia occurred as CAP, but the pneumococcal pneumonia in nursing-home and hospital cases possess different characteristics. NHAP is related to a bad prognosis and HAP is related to penicillin resistance.

Finally, we investigated the relationships between the critical events and A-DROP scores, CRP levels, and WBC counts. As shown in Table 5, 7, the critical events frequently occurred in patients with elevated A-DROP scores $(\geq 3$ points) and highly elevated CRP levels ( $\geq 30 \mathrm{mg} / \mathrm{dL})$. There 
Table 8. CRP Levels and Clinical Course

\begin{tabular}{|c|c|c|c|c|c|}
\hline $\mathrm{CRP}(\mathrm{mg} / \mathrm{dL})$ & Cases $(\%)$ & $\begin{array}{c}\text { Critical events } \\
(\%)\end{array}$ & p value * & $\begin{array}{c}\text { Duration of } \\
\text { therapy } \\
(\text { mean } \pm \text { S. D. })^{\dagger}\end{array}$ & $\mathrm{p}$ value ${ }^{\ddagger}$ \\
\hline$<10$ & $52(47.3 \%)$ & $2(3.8 \%)^{\S}$ & - & $11.8 \pm 3.8$ days & - \\
\hline $10-19.9$ & $28(25.5 \%)$ & $2(7.1 \%)$ & 0.61 & $13.3 \pm 5.7$ days & 0.32 \\
\hline $20-29.9$ & $23(21.0 \%)$ & $0(0 \%)$ & 1.00 & $18.2 \pm 11.2$ days & $<0.01$ \\
\hline$\geq 30$ & $7(6.4 \%)$ & $3(42.9 \%)^{\#}$ & 0.01 & $17.7 \pm 5.4$ days & $<0.01$ \\
\hline Total & 110 cases & 7 cases & & 104 cases & \\
\hline \multicolumn{6}{|c|}{$\begin{array}{l}{ }^{\dagger} \text { Four cases in which death occurred and two cases that transferred to other hospitals } \\
\text { before completing therapy were eliminated from analysis. } \\
\text { †p values vs. CRP }<10 \text { calculated by t-test. } \\
\text { \$D Death occurred in one case. } \\
\text { qDeath occurred in all cases }\end{array}$} \\
\hline
\end{tabular}

Table 9. WBC Counts and Clinical Course

\begin{tabular}{|c|c|c|c|c|c|}
\hline $\begin{array}{l}\text { WBC count } \\
(\text { cells } / \mu \mathrm{L})\end{array}$ & Cases $(\%)$ & $\begin{array}{c}\text { Critical events } \\
(\%)\end{array}$ & p value ${ }^{*}$ & $\begin{array}{c}\text { Duration of } \\
\text { therapy } \\
(\text { mean } \pm \text { S. D. })^{\dagger}\end{array}$ & p value ${ }^{\ddagger}$ \\
\hline$<4,000$ & $3(2.7 \%)$ & $2(66.7 \%)^{\S}$ & 0.02 & $21.3 \pm 5.8$ days & $<0.01$ \\
\hline $4,000-9,999$ & $43(39.1 \%)$ & $2(4.7 \%)^{\pi}$ & - & $12.8 \pm 4.1$ days & - \\
\hline $10,000-19,999$ & $53(48.2 \%)$ & $2(3.8 \%)^{\#}$ & 0.76 & $13.6 \pm 6.9$ days & 0.50 \\
\hline$\geq 20,000$ & $11(10.0 \%)$ & $1(9.1 \%)^{\&}$ & 0.87 & $18.5 \pm 13.1$ days & 0.02 \\
\hline Total & 110 cases & 7 cases & & 104 cases & \\
\hline 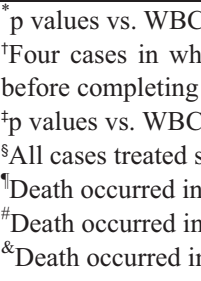 & $\begin{array}{l}\text { ounts of } 400 \\
\text { h death occu } \\
\text { erapy were e } \\
\text { ounts of } 400 \text { c } \\
\text { ceessfully. } \\
\text { ne case. } \\
\text { ll cases. } \\
\text { ne case. }\end{array}$ & $\begin{array}{l}9999 \text { cells } / \mu \mathrm{L} \\
\text { ed and two cas } \\
\text { ninated from an } \\
9999 \text { cells } / \mu \mathrm{L}\end{array}$ & $\begin{array}{l}\text { lculated } \\
\text { that tran } \\
\text { ysis. } \\
\text { lculated }\end{array}$ & $\begin{array}{l}\text { hi-square test. } \\
\text { red to other hosp } \\
\text { test. }\end{array}$ & \\
\hline
\end{tabular}

were no critical events in the low A-DROP score groups (0 or 1 points), but a few critical events were observed in the low CRP groups ( $<10 \mathrm{mg} / \mathrm{dL}$ and 10.0-19.9 mg/dL). Therefore, A-DROP scores are considered to be a better predictor of the critical events than CRP levels. We additionally performed multivariate analysis between five A-DROP consisting factors and the critical event. Only hypoxemia $(\mathrm{p}<0.01)$ and disturbance of consciousness $(\mathrm{p}<0.01)$ were extracted as independent factors that were related to the onset of critical event, but we should not deny the meaning of other factors (age, dehydration and hypopressure) in predicting the onset of critical event from this study. Further studies including pathogens other than pneumococcal pneumonia are needed to check the validity of A-DROP scores. In addition, decreased WBC counts $(<4,000$ cells $/ \mu \mathrm{L})$ can be regarded as a risk factor for the critical events because the incidence of the critical events was observed to be extremely high in this group $(66.6 \%$, Table 9$)$ though they were limited in number. There was a statistically significant correlation of prolonged periods of antibiotic therapy with CRP elevation (Table 8), but not with A-DROP scores. These observations suggest that the duration of therapy may be determined by CRP level rather than the disease severity, and that the duration of antibiotic therapy may not accurately reflect the disease severity. The duration of antimicrobial treatment seemed long, even in the group of A-DROP 0 points $(13.3 \pm 8.4$ days, Table 6). In most cases, antimicrobial treatment was ceased after normalization of CRP. Patient's age and comorbidity, such as preexisting respiratory disease $(32.4 \%$, Table 1), might affect the duration of therapy.

We analyzed the content of initial antibiotic therapy after visiting our hospital. Penicillin, cephalosporin, carbapenem, new quinolone, macrolide, and combination of carbapenem plus new quinolone were used in 45, 27, 19, 13, 1, 2 cases, respectively. Critical events occurred at 1 (2.2\%), 1 (3.7\%), $3(15.8 \%), 1(7.7 \%), 0(0 \%), 1(50.0 \%)$ case in penicillin, cephalosporin, carbapenem, new quinolone, macrolide, and combination of carbapenem plus new quinolone treated groups, respectively. The elevated incidence of critical events in the carbapenem-treated group was not likely due to the selection of antibiotics, because none of isolated $S$. pneumoniae in this study was IPM resistant (Table 5). It is not easy to discuss the regimen of antibiotics from this retrospective study.

In conclusion, we confirmed that A-DROP scores are useful for predicting the critical events in cases of pneumococcal pneumonia. Additionally our findings suggest that decreased WBC counts may be used as a prognostic factor since they were significantly correlated with the critical events despite the limited number of cases. CRP was not 
helpful for predicting the critical events, when compared to A-DROP scores. Furthermore, cross-sectional analysis of all pneumococcal pneumonia cases revealed that NHAP cases were at high risk for the critical events, and that PRSP was frequently isolated in HAP cases. These findings were obtained from retrospective analysis of 111 cases of pneumococcal pneumonia and provide important information in the management of patients with pneumococcal pneumonia. To assess our findings from the present study, prospective studies will be warranted not only for pneumococcal pneumonia, but also for pneumonia caused by other pathogens.

\section{The authors state that they have no Conflict of Interest (COI).}

Author contributions: S. Ikegame performed data collection and statistical analyses. K. Wakamatsu and H. Kumazoe participated in data collection. M. Kawasaki, M. Fujita, Y. Nakanishi, M. Harada, and A. Kajiki contributed to study organization.

Summary at a glance: We conducted a retrospective analysis of pneumococcal pneumonia cases. Critical events occurred more frequently in nursing home-acquired cases while hospitalacquired cases frequently developed penicillin resistance. Decreased WBC counts and elevated A-DROP scores were predictive of critical events. Future treatment of pneumococcal pneumonia should be modified based on these factors.

\section{References}

1. WHO. The world health report. 2003.

2. Vital Statistics; Trends in leading causes of death. Ministry of Health, Labour and Welfare, Tokyo, Japan 2010.

3. Saito A, Kohno S, Matsushima T, et al. Prospective multicenter study of the causative organisms of community-acquired pneumonia in adults in Japan. J Infect Chemother 12: 63-69, 2006.

4. Sopena N, Sabriá M, Pedro-Botet ML, et al. Prospective study of community-acquired pneumonia of bacterial etiology in adults. Eur J Clin Microbiol Infect Dis 18: 852-858, 1999.

5. Pinner RW, Teutsch SM, Simonsen L, et al. Trends in infectious diseases mortality in the United States. JAMA 275: 189-193, 1996.
6. American Thoracic Society. Infectious Diseases Society of America. Guidelines for the management of adults with hospitalacquired, ventilator-associated, and healthcare-associated pneumonia. Am J Respir Crit Care Med 171: 388-416, 2005.

7. Domínguez J, Galí N, Blanco S, et al. Detection of Streptococcus pneumoniae antigen by a rapid immunochromatographic assay in urine samples. Chest 119: 243-249, 2001.

8. Marcos MA, Jimenez de Anta MT, De La Bellacasa JP, et al. Rapid urinary antigen test for diagnosis of pneumococcal community-acquired pneumonia in adults. Eur Respir J 21: 209214, 2003.

9. Roson B, Fernandez-Sabe N, Carratala J, et al. Contribution of a urinary antigen assay (Binax NOW) to the early diagnosis of pneumococcal pneumonia. Clin Infect Dis 38: 222-226, 2004.

10. National Committee for Clinical Laboratory Standards. Performance standards for antimicrobial susceptibility testing: Ninth informational supplement. M100-S9. National Committee for Clinical Laboratory Standards 1999.

11. Committee for The Japanese Respiratory Society guidelines for the management of respiratory infections. Guidelines for the management of community acquired pneumonia in adults, revised edition. Respirology 11(Suppl 3): S79-S133, 2006.

12. Gutiérrez F, Masiá M, Rodríguez JC, et al. Evaluation of the immunochromatographic Binax NOW assay for detection of Streptococcus pneumoniae urinary antigen in a prospective study of community-acquired pneumonia in Spain. Clin Infect Dis 36: 286292, 2003.

13. El-Solh AA, Pietrantoni C, Bhat A, et al. Microbiology of severe aspiration pneumonia in institutionalized elderly. Am J Respir Crit Care Med 167: 1650-1654, 2003.

14. Fuse ET, Genma H, Sato M, et al. Evaluation of the usefulness of a rapid immunochromatographic membrane test to detect Streptococcus pneumoniae antigen in the early diagnosis of pneumococcal respiratory tract infections and the relationship to the severity of pneumonia. Nihon Kokyuki Gakkai Zasshi 46: 10-18, 2008 (in Japanese).

15. Depuydt P, Putman B, Benoit D, Buylaert W, De Paepe P. Nursing home residence is the main risk factor for increased mortality in healthcare-associated pneumonia. J Hosp Infect 77: 138-142, 2011.

16. Ishida $\mathrm{T}$, Maniwa $\mathrm{K}$, Kagioka $\mathrm{H}$, et al. Antimicrobial susceptibilities of Streptococcus pneumoniae isolated from adult patients with community-acquired pneumonia in Japan. Respirology 13: 240246, 2008.

(C) 2012 The Japanese Society of Internal Medicine http://www.naika.or.jp/imindex.html 